\title{
Simulation Results to Assess Effectiveness of Seals Made of Polyurethane for Hydraulic Drives of Machines
}

\author{
Mihail V. Drapalyuk \\ Department "Mechanization of Forestry and Machine \\ Design", \\ Voronezh State University of Forestry and Technologies \\ named after G. F. Morozov, \\ Timiryazeva 8, 394087 Voronezh, Russia \\ e-mail: Michael1@yandex.ru
}

Vladimir A. Zelikov

Department of "Organization of transportation and traffic safety"

Voronezh State University of Forestry and Technologies named after G. F. Morozov,

Lomonosova 112, 394087 Voronezh, Russia

e-mail: zelikov-vrn@mail.ru

\author{
Valerii I. Posmetev \\ Department "Production, repair and maintenance of \\ vehicles", \\ Voronezh State University of Forestry and Technologies \\ named after G. F. Morozov, \\ Timiryazeva 8, 394087 Voronezh, Russia \\ e-mail: posmetyev@mail.ru

\section{Vadim O. Nikonov} \\ Department "Production, repair and maintenance of \\ vehicles", \\ Voronezh State University of Forestry and Technologies \\ named after G. F. Morozov, \\ Timiryazeva 8, 394087 Voronezh, Russia \\ e-mai:1 8888nike8888@mail.ru
}

Eugene V. Shatalov

Department of "Organization of transportation and traffic safety"

Voronezh State University of Forestry and Technologies named after G. F. Morozov, Lomonosova 112, 394087 Voronezh, Russia

e-mail: opbd2017@mail.ru

\begin{abstract}
For optimization of geometrical parameters of the seal of movable joints of machines, a corresponding mathematical model is developed. For the computer implementation of the model, «Programme for simulation of piston seal" in Object Pascal in integrated programming environment Borland Delphi 7 is developed. To verify the adequacy of the estimated model, we conducted computer experiments on the bending of the sealing ring. Results of the analysis show that using new DCS design provides guaranteed increased efficiency of hydraulic drives of machines by reducing fluid leakage and response time of $\mathrm{HC}$ actuation, as well as improving the efficiency of hydraulic drives, especially in the conditions of operation of machines at lower temperatures of environment in spring and autumn periods.
\end{abstract}

Keywords- cars; exploitation; hydrauli; seal; simulati; efficiency; design.

\section{INTRODUCTION}

For the performance criteria of the dynamic connection seals (DCS) of hydraulic cylinders (HC) and other elements of the hydraulic drive of machines, we will accept tightness of seal and overall coefficient of performance (COP) of HC. Appropriateness of the selected criteria is justified as the latter ones are generalized. Therefore the most objective indicators of work of both the seals and HC are given in general [1-3].

According to the theory of tightness, reliability of DCS is determined by the flow rate of leakage of the power fluid and associated with the formation of lubricant film between contact surfaces during their mutual displacement. Numerous factors affecting the tightness and friction of DCS in the process of operation are subjected to significant change due to the ageing of the material and dependence of its mechanical properties on temperature, fatigue and wear of the destruction of the contacting surfaces, influence of vibrations and micro-deformations, alternating loads, contamination of the liquid, release of the wear products in a contact zone and dissolution of gases in the power fluid, etc [4-7]. Therefore, in analytical studies and engineering calculations of DCS, traditional empirical relationships are used with subsequent binding of experimental verification and correction of the obtained results [8].

For the generalized criterion of DCS tightness, we take specific linear leakage $Q_{n}$, expressed in $\mathrm{m}^{3} / \mathrm{m} \cdot \mathrm{cs}$ and specific linear leakage determined by the formula:

$$
Q_{n}=K Q_{a} / B=Q_{e} / B
$$

where $K=Q_{e} / Q_{a}-$ correction factor of leakage value $Q_{a}$, 
received analytically; $Q_{e}-$ leakage flow, found experimentally; $B$ - DCS perimeter.

For the foregoing reasons, the exact analytical determination of $Q_{a}$ leakage is extremely difficult. For this reason, the corresponding dependences, suggested by the number of researchers for the recognition of authors themselves, allow only a qualitative assessment of the flow rate of the leakage. In this regard, known empirical dependences in the direction of more consideration of the design and operation of DCS with respect to $\mathrm{HC}$ were updated. The resulting expression can be written in the form [9]:

$$
Q_{a}=n_{x} \frac{1}{2} \pi D_{y} L_{y}\left(\sqrt{\frac{\mu_{1} v_{1} \Delta P_{1}}{p_{k 1} l_{k 1}}}-\sqrt{\frac{\mu_{2} v_{2} \Delta P_{2}}{p_{k 2} l_{k 2}}}\right),
$$

where $n_{x}$ - double-stroke number of piston; $D_{y}$ - diameter of sealed surface; $\pi \cdot D_{y}=B$ - perimeter of sealing; $L_{y}$ - piston strike; $\mu$-dynamic-viscosity coefficient of power fluid; $v$ - piston speed; $\Delta P-$ differential pressure $\left(P_{c}-P_{p}\right)$ in cylinder $P_{c}$ and piston $P_{p}$ cavities; $p_{c}-$ specific contact force in the sealing; $l_{c}-$ contact width of sealing element with a counterbody.

In the formula (2), the first radical expression in the brackets defines the thickness of the film of lubricant when running, and the second one - during idle run of the HC piston or accumulator piston. The difference between the values of the thicknesses of these films determines the presence (absence) and the continuity of the film of lubricant. For this reason, between the contacted surfaces of the DCS there are the following types of friction: dry, boundary-dry, boundary, boundary-liquid and that of liquid. In the first three cases during the work of DCS HC of unilateral action, there is a "pumping" effect in varying degrees, leading to air excess in the system and power fluid saturation with it, which ultimately worsens the operation of the hydraulic drive of PM. At the same time during the liquid friction, a significant amount of leakage of the power liquid is possible, also reducing the efficiency of the hydraulic drive. Therefore, the most appropriate type of friction that should be assigned during DCS design is boundary-liquid one, which has a combination of minimal friction and little leakage of the power fluid.

The analysis of influence of constructive and operating parameters of DCS on the leakage shows that the value of the latter one is proportional to $\mu, \Delta P, v$ and inversely proportional to $p_{c}, l_{c}$. It is obvious that in DCS design, only independent parameters $\mu, p_{c}$ and $l_{c}$ are variable, of which the first one must be accepted as the lowest and two others - the highest one of the possible values. It is also important to note that the dependence of the viscosity $\mu$ of the pressure will be different for different temperatures and types of fluid in the general case; it is expressed by an exponential function. However, for practical calculations according to the expression (2) in the pressure range of $0-40 \mathrm{MPa}$, we can assume that the viscosity of the fluid linearly depends on the pressure. This assumption allows us to use the empirical formula:

$$
\mu=\mu_{0}(1+a \Delta P)
$$

where $\mu_{0}$ - dynamic-viscosity coefficient at atmospheric pressure; $a$-coefficient depending on the type of power fluid
$\mathrm{MPa}$ (at $v_{50}<15 \mathrm{cSt}, a=0.002$; at $v_{50}>15 \mathrm{cSt}, a=0.003$ ).

During estimated calculations on expression (2), the product $p_{c} \cdot l_{c}$ can be replaced by contact pressure $p$, defined for elastic DCS with the empirical expressions:

$$
p=p_{\varepsilon}+\kappa \Delta P \text {, }
$$

where $p_{\varepsilon}-$ contact pressure, made during mounting $\left(p_{\varepsilon}=\right.$ $0.02-0.04 \mathrm{MPa}-$ for sealing rings; $p_{\varepsilon}=0.2-0.5 \mathrm{MPa}-$ for round section rings); $\kappa-$ influence coefficient of DCS type $(\kappa=0.6-$ 0.7 - for sealing rings; $\kappa=0.8-0.9$ - for round section rings).

Overall efficiency $\eta_{o}$ HC DM will be defined from expression:

$$
\eta_{o}=\eta_{m} \cdot v_{\partial} / v t
$$

where $\eta_{m}$ - coefficient of mechanical efficiency HC; $v_{\partial}$ and $v_{t,}-$ real and theoretical velocities of piston movement.

Coefficient $\eta_{m}$ for $\mathrm{HC}$ of single action in the general case is calculated by the formula:

$$
\eta_{m}=\frac{4 \cdot F_{\partial}}{\pi\left[D_{y}^{2} P_{c}-\left(D_{y}^{2}-d_{p}^{2}\right) P_{p}\right]},
$$

where $F_{\partial}$ - measured value of force made by $\mathrm{HC} ; d_{p}-$ piston diameter.

As far as $\mathrm{HC}$ has $P_{p}=0$, the final expression for determining $\eta_{m}$ will be:

$$
\eta_{m}=\frac{4 \cdot F_{\partial}}{\pi D_{y}^{2} P_{c}}
$$

Thus, for more objective quantification of new DCS according to expressions (1) ... (4), it is necessary to determine experimentally the amount $Q_{e}$ of fluid leakage flow, the actual values of speed $v_{\partial}$, piston movements and efforts $F_{\partial}$ for each of the investigated DSC.

\section{RESULTS}

For optimization of geometrical parameters of the seal of movable joints of machines, the corresponding mathematical model is developed. The model is based on the method of particle dynamics. We decided to use a two-dimensional version of the model (longitudinal cross-section of the cylinder), since the problem has axial symmetry. Thus the radial distortion of this approximation is negligible, since the thickness of the sealing ring is significantly smaller than the inner diameter of the cylinder. In the framework of the sealing ring design, the metal parts of the hydraulic cylinder and the power fluid are represented by many elements-circles of a small diameter of $0.2 \mathrm{~mm}$ order (hereinafter elements) (Fig. $1)$. 


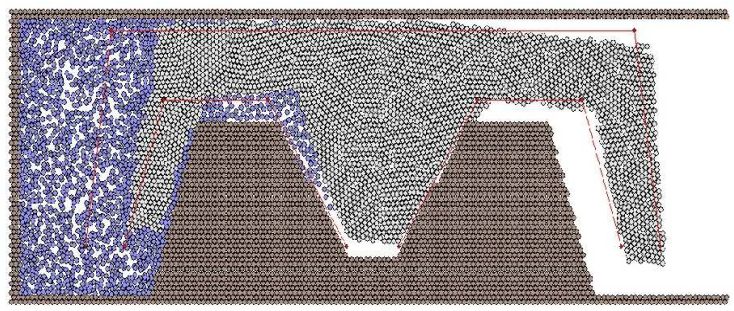

Fig. 1. Sealing ring of metal parts of hydraulic cylinder and power fluid as aggregate of sets of elements of round form

Main mechanical characteristics of the elements in the framework of particle dynamics (mass, stiffness coefficient, viscous friction, constraints of interactions) are based on the table values of parameters of the respective environments density, elastic modulus, friction coefficient and strength tensile limit. The movement of the elements is described by the differential equations of the second order in Newtonian form, which are solved by Runge-Kutta numerical method of the second order.

The sealing ring has complex cross-sectional shape and is characterized by eight geometric parameters $l_{\mathrm{a}}, l_{\mathrm{b}}, l_{\mathrm{c}}, l_{\mathrm{d}}, l_{\mathrm{e}}, l_{\mathrm{f}}$, $l_{\mathrm{g}}, l_{\mathrm{h}}$ (Fig. 2)

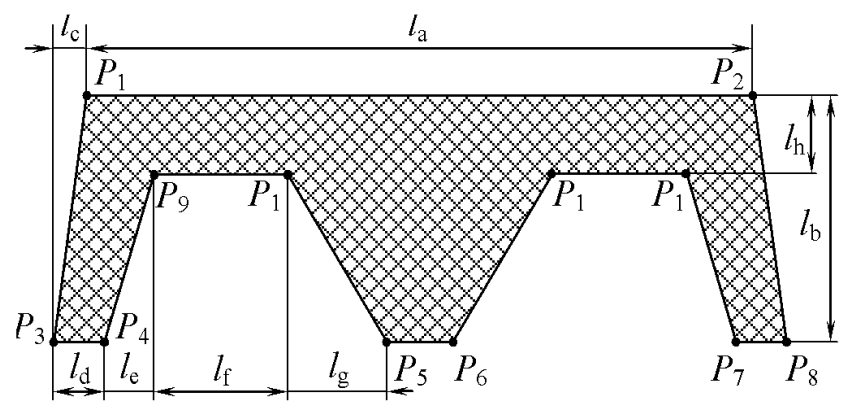

Fig. 2. A calculation scheme for the task in the model of the geometric area, representing a sealing ring section

The geometric area, giving a cross section of the investigated sealing ring, is limited by broken line $P_{1} P_{2} P_{8} P_{7} P_{12} P_{11} P_{6} P_{5} P_{10} P_{9} P_{4} P_{3}$. Calculation of coordinates of points in the model is made by the following formulas:

$$
\begin{aligned}
& x_{P 1}=\frac{L_{x}}{2}-\frac{c_{a}}{2} ; y_{P 1}=\frac{L_{y}}{2}+\frac{c_{b}}{2} ; \\
& x_{P 2}=\frac{L_{x}}{2}+\frac{c_{a}}{2} ; y_{P 2}=\frac{L_{y}}{2}+\frac{c_{b}}{2} ; \\
& x_{P 3}=\frac{L_{x}}{2}-\frac{c_{a}}{2}-c_{c} ; y_{P 3}=\frac{L_{y}}{2}-\frac{c_{b}}{2} ; \\
& x_{P 4}=\frac{L_{x}}{2}-\frac{c_{a}}{2}-c_{c}+c_{d} ; \\
& y_{P 4}=\frac{L_{y}}{2}-\frac{c_{b}}{2} ;
\end{aligned}
$$

$$
\begin{aligned}
& x_{P 5}=\frac{L_{x}}{2}-\frac{c_{a}}{2}-c_{c}+c_{d}+c_{e}+c_{f}+c_{g} ; \\
& y_{P 5}=\frac{L_{y}}{2}-\frac{c_{b}}{2} ; \\
& x_{P 6}=\frac{L_{x}}{2}+\frac{c_{a}}{2}+c_{c}-c_{d}-c_{e}-c_{f}-c_{g} ; \\
& y_{P 5}=\frac{L_{y}}{2}-\frac{c_{b}}{2} ; \\
& x_{P 7}=\frac{L_{x}}{2}+\frac{c_{a}}{2}+c_{c}-c_{d} ; \\
& y_{P 12}=\frac{L_{y}}{2}+\frac{c_{b}}{2}-c_{h}, \\
& y_{P 7}=\frac{c_{b}}{2} ; \\
& y_{P 10}=\frac{L_{y}}{2}+\frac{L_{x}}{2}+c_{h} ; \\
& x_{P 8}=\frac{L_{x}}{2}+\frac{c_{a}}{2}+c_{c} ; \\
& x_{P 8}=\frac{L_{y}}{2}-\frac{c_{b}}{2}-c_{b} \\
& x_{P} \\
& x_{P}
\end{aligned}
$$

where $x_{P i}$ and $y_{P i}-$ Cartesian ordinates of $i$-th of reference point $P_{\mathrm{i}} ; L_{\mathrm{x}}$ and $L_{\mathrm{y}}-$ dimensions of rectangular model space, in which elements can move.

Before the computer model experiment is conducted, filling of the geometrical area that corresponds to the plane of sealing ring is made with elastic material elements (Fig. 3). To 
do this, the first random close packing of 6800 elements (Fig. $3, a)$ is formed, covering the geometric area. Forming the random close packing is made by the same method of particle dynamics: initially, elements are distributed randomly on a rectangular area (using the random number generator with software implementation). After that, the integration of the equations of motion elements is made within $0.5 \mathrm{~s}$ with the result; those elements, seeking to sit in such way that the distances between adjacent items were roughly the same.

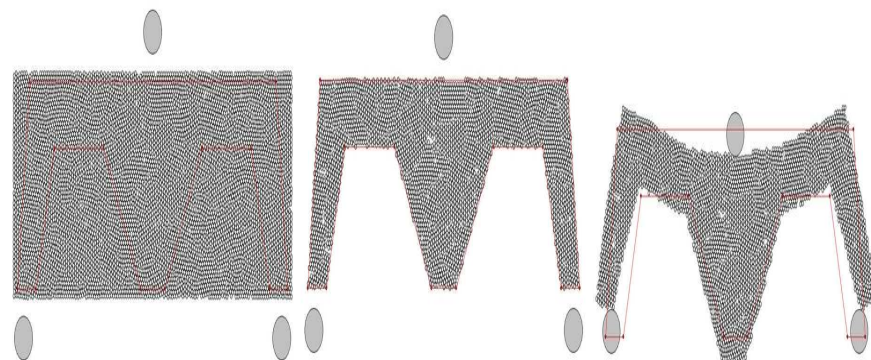

$a$ - initial rectangular area, with a random close packing elements of an elastic material;

$b$-initial state of the cuff; $c$ - bend the cuff as a result of round-shaped indenters

Fig. 3. Mechanical testing of the cuff in order to verify the elasticity and strength

Then the sealing ring is "cut" from a rectangular area: all the elements that do not fall within the region bounded by the thin line in Figure 2 are removed. Elements with coordinates $\left(x_{i}, y_{i}\right)$ are considered as not belonging to the sealing ring, if the following condition is created (5):

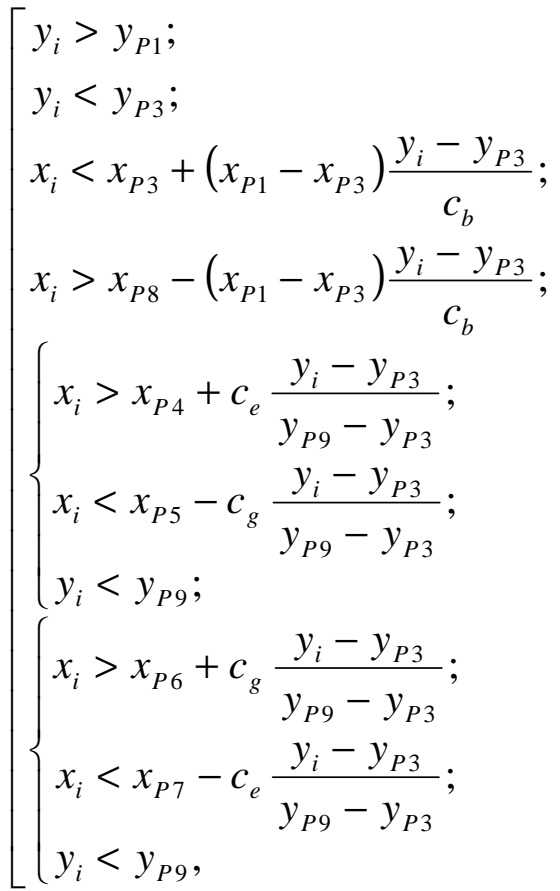

where symbol «[» means what at least one of the listed symbol conditions should be done; symbol «\{»- that all of these under the symbol conditions must simultaneously run.

For the computer implementation of the model,
«Programme for simulation of piston seal" in Object Pascal in integrated programming environment Borland Delphi 7 is developed. To verify the adequacy of the estimated model, we conducted computer experiments on the bending of the sealing ring. The sealing ring was applied with external force from three indentures of circular cross-section, moving at a low speed in the direction of the sealing ring. As a result, the sealing ring is gradually curved.

It was discovered that when using traditional materials for rubber used in sealing technology in the model, the sealing ring in the model is not destroyed even with significant bends and returns to its original state after removing external force effects (after removal of indenture to their original positions) [10].

The dveloped simulation model of the movable joints seals of forest machine hydraulic drives of new design allows you to explore various modes of the seal and optimize the construction parameters of the sealing ring and the snap ring of the hydraulic cylinder piston.

\section{DISCUSSION}

Analysis of the results of the simulation also showed that differential pressure of working fluid on the left and on the right strongly influences the shape of the sealing ring and, consequently, the effectiveness of seal. At equal pressures on both sides of the sealing ring, it is basically in symmetric condition (Fig. 4). At low differential pressure (less than 1 $\mathrm{MPa}$ ), the sealing ring is pressed against the inner surface of $\mathrm{HC}$ and prevents power fluid infiltration. Although the degree of resistance is low, the pressure of the power fluid is also small enough to cause significant chocking in remaining clearance. In case of large pressure difference $(10-20 \mathrm{MPa})$, the sealing ring not only stronger comes to the inner surface of $\mathrm{HC}$, but at the same time it is pressed against the beveled piston wall, which causes wedging of the middle part of the sealing ring and a reliable seal at both top and bottom parts of the sealing ring [11].

At very high pressures in the hydraulic drive (40 to 60 $\mathrm{MPa}$ ), elasticity of compression of the sealing ring, made from traditional materials, can lead to its srumple and(or) destruction (Fig. 4, d). Therefore, sealing rings of high pressure $\mathrm{HC}$ must be made of elastic materials with a high modulus of elasticity and protect them from destruction with the help of additional elements of highly durable polymer materials. In addition, to eliminate the crushing by changing geometric parameters of the sealing ring (a more massive sealing ring as a whole, or a smaller size of the clearance between the thrust metal ring and the inner surface of $\mathrm{HC}$ ).

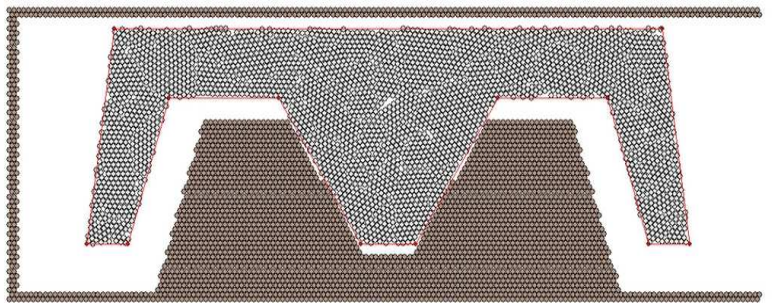




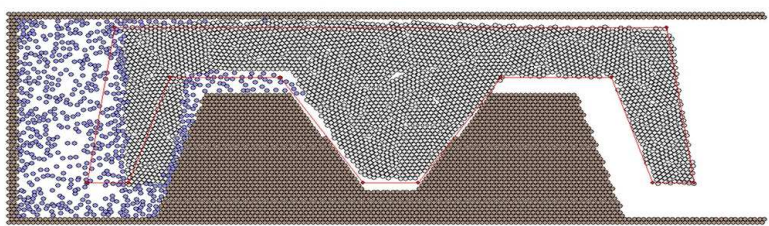

$\mathrm{b}$

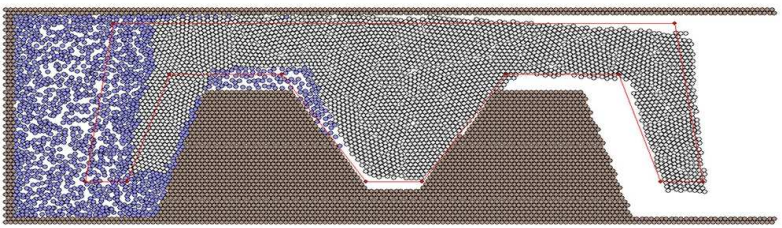

$\mathrm{c}$

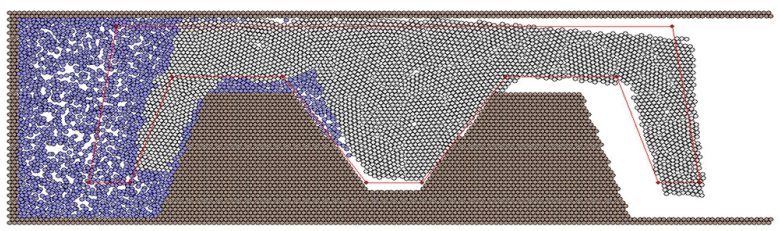

d

$\mathrm{a}$ - basic condition of sealing ring in case of equal pressure from both sides of piston; condition of sealing ring in measuring pressure in piston area; $b-1$

$\mathrm{MPa} ; \mathrm{c}-$ start of deformation of sealing ring at pressure of $10 \mathrm{MPa} ; \mathrm{d}-$ crumple of sealing ring at pressure of $50 \mathrm{MPa}$

Fig. 4. Sealing ring condition at different pressures of power fluid

Simulation results and laboratory and field experiments of investigated DCS, obtained for their three basic parameters linear leakage $Q_{l}$ of power fluid, response time $t_{r}$ of actuation and overall efficiency $\eta_{o}$ of $\mathrm{HC}$, are represented in Figure 5. Analysis of obtained data indicates the following.

Values of all three fixed parameters largely depend on changes in the ambient temperature and especially in the field of negative $0 \ldots-10$ and low $0 \ldots+10^{0} \mathrm{C}$ temperatures. The intensity of a decrease of parameters values in the direction of deterioration of $\mathrm{HC}$ operation is about 1.5-2.0 times higher than at $T>10^{\circ} \mathrm{C}$. This disadvantage is hard to handle in both DCSs and it is common to all types of DCS, especially in spring and autumn periods, which should be considered by machine operators in the operation of FM on forest sites at low ambient temperatures.

Leakage $Q_{l}$ of power fluid at new DCS does not exceed the leakage of any standard seal (Fig. 5, $a$ ), which evidences about its good integrity in the entire range of possible ambient temperatures

Response time $t_{r}$ of HC actuation with new DCS is on average by 1.6 lowers than with standard DCS (Fig. 5, b). Thanks to this, both hydraulic and working bodies of forest machines are considerably less exposed to dynamic loads. Consequently, response time of $\mathrm{HC}$ actuation, as well as through the use of the sealing ring, made of polyurethane in DCS with a significantly lower friction coefficient, is reduced compared with the standard rubber sealing ring, the efficiency of new DCS has increased twice on average (Fig. 5, c).
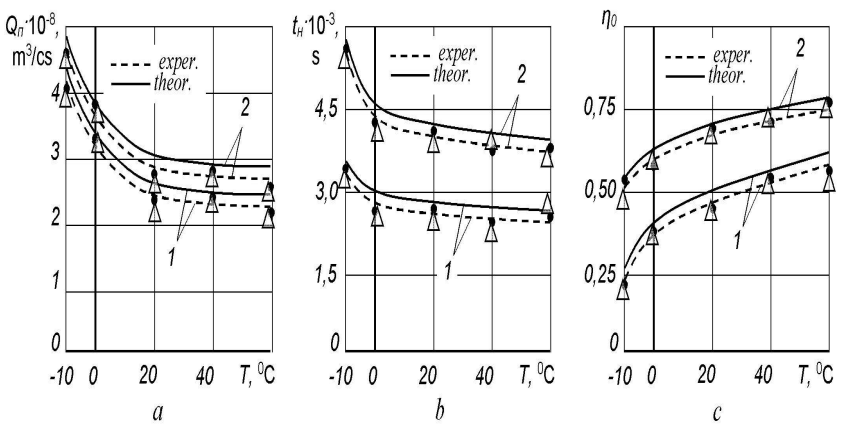

1 and 2 - curves of new and standard DCS, respectively

Fig. 5. Dependences of specific line leakage of power fluid Q1 on speed response tr of hydraulic cylinder actuation; overall efficiency $\eta$ o of hydraulic cylinder on $\mathrm{T}$; temperature of power fluid for researched seal elements

\section{CONCLUSION}

Thus, the results of the analysis show that using new DCS design provides guaranteed increased efficiency of hydraulic drives of forest machines by reducing fluid leakage and response time of HC actuation, as well as improving the efficiency of hydraulic drives, especially in the conditions of operation of machines at lower temperatures of environment in spring and autumn periods.

\section{References}

[1] Valeriy I. Posmetyev, Vladimir A. Zelikov, Michael V. Drapalyuk, Margarita A. Latysheva and Evgeniy V., Shatalov Substantiation and evaluation of effectiveness of perspective constructions of forest tractors ancillary equipment, ARPN Journal of Engineering and Applied Sciences, Vol. 11 № 3, pp. 1840-1855, 2016.

[2] M. Osman Abdalla, Nagarajan T., Fakhruldin M. Hashim / International Journal of Engineering Research and Applications (IJERA) ISSN: 22489622 www.ijera.com Vol. 3, Issue 1, January-February 2013, pp. 001007.

[3] T. E. Beacham, F. H. Towler, "Rotary, oscillating \& reciprocating seals", Industrial Lubrication and Tribology, Vol. 2 Iss : 3, pp. 12-15, 1950.

[4] V. A. Zelikov, V. I. Posmetiev, M. A. Latysheva, Substantiation Based on Simulation Modeling of Hitch for Tillage Tools Parameters, World Applied Sciences Journal, № 30(4), pp. 486-492, 2014.

[5] R. Vishnu Ramesh Kumar, V. Varun Sagar, P. Vishnuram, N. Hari Prasanth, Optimization of Hydraulic Oil Seal in Earth Movers, International Journal of Science and Research (IJSR), Volume 5 Issue 2, pp. 2156-2162, February 2016.

[6] B. S. Nau, An historical review of studies of polymeric seals in reciprocating hydraulic systems, Proceedings-institution of mechanical engineers part $\mathrm{j}$ journal of engineering tribology, Vol. 213, 3, pp. 215226, seals by Mechanical engineering publications limited, 1999.

[7] M. Jocanovic, D. Sevic, V. Karanovic, I. Beker, S. Dudic, Increased efficiency of hydraulic systems through reliability theory and monitoring of system operating parameters, Stroj. Vestnik-J. Mech. Eng., Vol. 58, pp. 281-288, 2012.

[8] Y. Yorozu, M. Hirano, K. Oka, and Y. Tagawa, "Electron spectroscopy studies on magneto-optical media and plastic substrate interface," IEEE Transl. J. Magn. Japan, vol. 2, pp. 740-741, August 1987 [Digests 9th Annual Conf. Magnetics Japan, p. 301, 1982]. 
[9] L.A. Kondakov, Seals of hydraulic systems: teaching aid, M.: Mechanical Engineering, 1972.

[10] M. Young, The Technical Writer's Handbook. Mill Valley, CA: University Science, 1989.
[11] H. Course, Development of an hydraulic seal, Design engineering, Vol. 5, pp. 99-100, 1977. 